\title{
Assessment of Knowledge, Attitude, Practice and Determinants of VCT Utilization for HIVIAIDS Among Ambo University Students, West Shoa Zone, Oromia Region, Ethiopia: Cross Sectional Study
}

\author{
Getabalew Endazenaw Bekele, Ketema Kensa, Mitswat Abebe, Kokeb T/Mariam \\ Ambo University, College of Medicine and Health Sciences, Department of Nursing \& Midwifery, Ambo, Ethiopia
}

Email address:

eyobgetabalew@gmail.com (G. E. Bekele),zem_get@yahoo.com (G. E. Bekele)

To cite this article:

Getabalew Endazenaw Bekele, Ketema Kensa, Mitswat Abebe, Kokeb T/Mariam. Assessment of Knowledge, Attitude, Practice and Determinants of VCT Utilization for HIV/AIDS Among Ambo University Students, West Shoa Zone, Oromia Region, Ethiopia: Cross Sectional Study. Science Journal of Public Health. Vol. 3, No. 2, 2015, pp. 259-264. doi: 10.11648/j.sjph.20150302.25

\begin{abstract}
Voluntary HIV counseling and Testing (VCT) is a process by which an individual undergoes counseling to enable informed about HIV status. The provision of voluntary HIV counseling and testing (VCT) is an important part of any national prevention program. This study tried to assess the knowledge, attitude and practice determinants of voluntary counseling and testing utilization for HIV/AIDS of Ambo University students. Descriptive cross-sectional study design was implemented with multistage sampling apply in the study. There were 93.2 response rates in the study. Three hundred eight nine (51.9\%) of the respondents have sexual experience, or ever had sexual intercourse; out of which 288 (38.4\%) were males and the rest $101(13.5 \%)$ were females. Among the total participants 504(67.2\%) were used VCT services but 246(32.8\%) were never used VCT service. Regarding the location of VCT service recommended, health facility, school and youth club were recommended by 337(49.7\%), 310(41.3) and 219(29.2\%) of the students respectively. There were different determinants those have statistical association with VCT service utilization include sex, batch, sexual exposure and knowledge of the students about VCT. Even though most of the students have adequate knowledge, attitude and practice still there is a gap in the participants KAP towards VCT utilization due to different determinants like sex, sexual exposure and their level of education (batch).
\end{abstract}

Keywords: Voluntary HIV Counseling and Testing, Knowledge, Attitude, Practice

\section{Introduction}

Voluntary HIV counseling and Testing (VCT) is a process by which an individual undergoes counseling to enable him/her to make informed choice about being tested for HIV. It consists of pre-test and post-test counseling. HIV/AIDS counseling is a confidential communication between a client and care provider aimed at enabling the client to cope with stress and to take personal decisions relating to HIV/AIDS. The goal of VCT services is to enable individual and couples to learn their results voluntarily in to a setting in which confidentiality is strictly maintained. ${ }^{1,2,3,4,5,6}$

Access to VCT is a key for successfully implementing anti-retroviral treatment and avoiding re-infection and transmission through behavioral changes. Voluntary counseling and testing (VCT) also entry points for successful implementation of prevention, care and support services among both HIV negative and positive individuals. Effective knowledge of HIV status is critical to expanding access to HIV treatment, care and support timely and in offering people living with HIV an opportunity to receive information and tools to prevent transmission to others. Voluntary counseling and testing (VCT) is recognized as one of the few potentially effective and affordable methods for reducing the transmission of HIV in developing countries. ${ }^{7,8,9}$

Various studies have shown that acceptance of VCT is influenced by knowledge about HIV transmission and prevention, knowledge about VCT, positive attitudes towards HIV testing and self-perceived risk of HIV infection. A study on HIV voluntary counseling and testing service preference in rural Malawi among 868 women aged 15-34 and 648 men aged 20-44 revealed that knowledge about HIV/AIDS 
prevention, knowing someone with AIDS, knowing the locations of a test site and perceived risk of HIV infection all had consistently significant association with VCT use for men and women. ${ }^{10}$

A study done in Addis Ababa showed that all 640 (100\%) of study subjects agreed that pre-marital VCT is important. Prevention of heterosexual HIV transmission (93.6\%), planning for the future $(87.5 \%)$ and planning to have a child $(86.3 \%)$ were some of the reasons given for VCT utilization by pre-marital VCT users. Male subjects who had no sexual contact with their fiancées and males with a history of another prior marriage were significantly and more likely to be associated with VCT utilization $[\mathrm{OR}=9.62(1.23-75.2)]$ and $\left[\mathrm{OR}=(0.2=2.96(1.22-7.18)]\right.$ respectively ${ }^{11}$.

A study in Northwest Ethiopia Gondar on assessment of determinants on VCT acceptance revealed that those who had self-perceived risk and knowledge of HIV/AIDS prevention methods were more likely to accept VCT than their counterparts respectively. ${ }^{12}$

According to research done at Mekele university among 425 university students, among the respondents about $35.1 \%$ preferred the VCT service to be given in youth clubs and about the $84(20 \%)$ of the respondents believed that if someone has tested HIV positive he/she should have to teach others about the condition. ${ }^{13}$

According to the findings of the research done in Bahir Dar University revealed males were 1.8 times more likely to accept VCT service as compared to female students. Regarding the years of study first, second, and third year students were more likely to test than fourth year students. Students who had high knowledge about HIV and VCT were 2.4 times more likely tested than their counter part as well as those who had positive attitude toward VCT more likely tested than those had negative attitude. In addition students who perceived themselves at high risk of HIV were 6.3 times more likely to be tested than those who did not ${ }^{14}$.

The overall conditions of the utilization of VCT service in Ethiopia calls for scaled up and organized activities carried out to improve the service based on available evidence. Beside this, university students are at risk of HIV because multifactorial reason including high risk for peer pressure .Thus, this study planned to assess knowledge, attitude, practice towards VCT and factors associated to utilization of VCT service among students in Ambo University, Ambo, Ethiopia.

\section{Method and Materials}

Descriptive cross sectional study design was performed to determine knowledge, attitude and practice and determinant factors of VCT utilization for HIV among Ambo university students using self-administered questionnaire from January to June 2012.Multi stage sampling technique used to select study participants from all first, second and third year regular and CEP students. sample size was determined by using single population proportion sample size calculation formula with assumption of the prevalence of VCT utilization $50 \%$,
$95 \%$ confidence interval and $5 \%$ of margin error and, multiply with design effect 2 and $10 \%$ of none response rate hence, the sample would be 805 .The data analysis starting from collecting and Epi-info and SPSS were used to analyze the collected data. Ethical clearance was obtained from Ambo University College of medicine and Health science by writing the official letter and permission to the institution required. All Ambo University students who participated in the study were interviewed for their willingness to participate in the study.

\section{Result}

A total number of 750 respondents were participated (93.2 response rate) in the study. Among the total participants 561(74.8\%) were male students whereas $189(25.2 \%)$ were females. The mean age (+SD) of respondents was 21.22 years $(+3.07)$ (median 22 years). The majority of the students $675(90 \%)$ were single in their marital status and 411(54.8\%) orthodox, $174(23.2 \%)$ were protestant. Regarding the respondents batch 266(35.5\%), 299(39.9\%) 124(24.6\%) were first year, second year and third year respectively.

Three hundred eight nine $(51.9 \%)$ of the respondents have sexual experience, or ever had sexual intercourse; out of which 288 (38.4\%) were males and the rest $101(13.5 \%)$ were females. Age at first sexual contact ranges from 14-44 years of age and the majority of them being 14-19 years of age at their first experience.

With regard to protecting oneself from HIV infection, 352 $(46.9 \%)$ of the students claim that they were abstaining from sex, out of which 261(34.8) were males and the rest, $91(12.1 \%)$ were females. One hundred ninety five $(26.0 \%)$ of them were being faithful to partners, among these $140(18.7 \%)$ were males and 55(7.3\%) were females and 203(27.2\%) of the total students were using condoms for protection themselves from HIV, of which $160(21.4 \%)$ were males and the rest were female students. Regarding their current sexual partners $332(44.3 \%)$ of the students had sexual partner(s) of which $238(31.7 \%)$ were males and the rest, 94(12.5\%) were females (Table 1).

Three hundred thirty $(44.0 \%)$ of the students said that they know three modes of HIV transmission, 84 (11.2\%) said they know two ways, $223(29.7 \%)$ of them claim to know four ways of HIV transmission and $102(13.7 \%)$ of the students said that they know five and above method of HIV transmission. (Fig 1)

The major source of information about HIV/AIDS, identified by the students were school and radio/TV $366(48.8 \%)$ followed by school only and radio/TV only which accounts $172(22.9 \%)$ and $158(21.1 \%)$ respectively (fig 2).

Coming to the students' information regarding VCT services, $705(94 \%)$ of them know about or have heard of VCT services from different sources and the rest of the participants never hear any information about VCT. Six hundred sixty seven $(88.9 \%)$ of the students have said that VCT is an important for prevention of HIV transmission and 
the rest $83(11.1 \%)$ of them believed that VCT has no importance in the prevention and controlling HIV transmission as well as $714(95.2 \%)$ of the students have agreed that counseling is important for HIV testing. (Table 3)

Among those reported that VCT is important for HIV prevention, the majorities, $54.1 \%$ of them have agreed that VCT is important to change behavior of the person and preventing HIV infection, $44 \%$ of them said it is also used to know HIV status, $37.9 \%$ of the students also believed that VCT is used to protect others from getting infected, and $31.6 \%$ of them said it is important to get psychological support. Regarding the beneficiary from being tested, 297 (39.5\%) of the students were believed that HIV negative individuals are beneficiary. (Table 2)

The majority, $465(62.0 \%)$ of the students were agreed that everybody who is sexually active needs VCT service. But $172(23.0 \%)$ of them believed only those suspected by medical personnel need VCT service. Seven hundred ten $(94.7 \%)$ of the students were knew the location of VCT service area. Among those who know the location of VCT service area, $481(64.1 \%)$ of them lived near to the VCT service area whereas $188(25.1 \%)$ were lived far away from VCT service area. Regarding the location of VCT service, $606(80.8 \%)$ and $109(14.6 \%)$ of the students were identified in health facilities and youth club or association respectively. Among the total participants 504(67.2\%) were used VCT services but 246(32.8\%) were never used VCT service. Among those who were tested for HIV, $48 \%$ of them to know their HIV status, 9\% had multiple sexual partners and $7 \%$ had unprotected sex as their main reasons.

Regarding the location of VCT service recommended, health facility, school and youth club were recommended by $337(49.7 \%), 310(41.3)$ and $219(29.2 \%)$ of the students respectively. Five hundred forty seven $(73.0 \%)$ of the students were preferred trained health personnel for counselor for adolescents, 264(35.2\%) and 188(25.1\%) of the students were preferred trained youth (peer group and religious leaders as counselor for adolescent respectively.

Regarding HIV test result disclosure, the majority, $222(29.6 \%)$ of the students did not to disclose to anybody, whereas $175(23.3 \%), 132(17.6 \%)$ and $54(7.0 \%)$ the students were disclosed to their family, friends and health professionals respectively. Six hundred fifty five $(87.3 \%)$ of the students were ever seen a person with HIV positive result and $615(82.2 \%)$ of them were also ever seen a patient with AIDS. Five hundred twenty seven $(70.3 \%)$ of the participants were believed that living with HIV positive persons is safe. Regarding the precaution taken while living with HIV positive person, 373(49.7\%), 180(24.0\%) and 172(22.9\%) of the students were mentioned avoid body fluid contact; avoid external body contact and separate bed and eating utility materials as precaution methods respectively.(Table 2)

Table 1. Sexual history profile of the students in Ambo University, March, 2012.

\begin{tabular}{|c|c|c|c|c|}
\hline Characteristics & & Male N, (\%) & Female N, (\%) & Total \\
\hline \multirow[t]{2}{*}{ Sexual experience } & Yes & $288(38.4)$ & $101(13.5)$ & $389(51.9)$ \\
\hline & No & $273(36.4)$ & $88(11.7)$ & $359(47.9)$ \\
\hline \multirow[t]{2}{*}{ Age at first sexual contact } & $14-19$ & $239(61.2)$ & $93(24.9)$ & $332(86.1)$ \\
\hline & $\geq 20$ & $49(12.6)$ & $9(2.3)$ & $58(14.9)$ \\
\hline \multirow[t]{2}{*}{ Having sexual partner currently } & Yes & $238(31.7)$ & $94(12.5)$ & $332(44.3)$ \\
\hline & No & $323(43.1)$ & $95(12.7)$ & $418(55.7)$ \\
\hline \multirow[t]{3}{*}{ Method of protection of HIV } & Abstinence & $261(34.8)$ & $91(12.1)$ & $352(46.9)$ \\
\hline & Faithfulness & $140(18.7)$ & $55(7.3)$ & $195(26.0)$ \\
\hline & Using condoms & $160(21.4)$ & $43(5.8)$ & $203(27.2)$ \\
\hline
\end{tabular}

Table 2. VCT service knowledge, attitude and practice of the students in Ambo University, March, 2012.

\begin{tabular}{|c|c|c|}
\hline Characteristics & Frequency $(\mathrm{N}=750)$ & Percentage $(\%)$ \\
\hline \multicolumn{3}{|l|}{ Have you ever heard about VCT services? } \\
\hline Yes & 705 & 94.0 \\
\hline No & 45 & 6.0 \\
\hline \multicolumn{3}{|c|}{ Is VCT important for prevention of HIV transmission? } \\
\hline Yes & 667 & 88.9 \\
\hline No & 83 & 11.1 \\
\hline Have no use & 83 & 11.1 \\
\hline To know HIV status & 330 & 44.0 \\
\hline To change behavior and prevent HIV infection & 406 & 54.1 \\
\hline To protect others from being infected & 284 & 37.1 \\
\hline To get psychological support & 237 & 31.6 \\
\hline To get information about HIV/AIDS & 261 & 34.8 \\
\hline Who do you think benefits from being tested? & & \\
\hline
\end{tabular}




\begin{tabular}{lll}
\hline Characteristics & Frequency (N=750) & Percentage (\%) \\
\hline HIV positive individuals & 239 & 31.9 \\
HIV negative individuals & 297 & 39.5 \\
Both HIV positive and negative individuals & 214 & 28.6 \\
Do you think counseling is important for HIV testing? & & 95.2 \\
Yes & 714 & 4.8 \\
No & 36 & 41.5 \\
Who do you think needs to be tested?* & & 29.2 \\
High-risk groups & 311 & 62.0 \\
Those to be married & 219 & 23.0 \\
Everybody who is sexually active & 465 & 4.4 \\
Only those suspected by medical personnel & 172 & 94.7 \\
Others & 33 & 5.3 \\
Do you know where you can get the service? & & \\
Yes & 710 & 64.1 \\
No & 40 & 25.1 \\
Is the service near your area or far? & & 5.5 \\
Near & 481 & 67.2 \\
Far & 188 & 32.8 \\
Very far & 41 & \\
Yes & & \\
No & 504 & 246 \\
\hline
\end{tabular}

* Percentages do not add up to 100 , because the questions are with possible multiple answers

Table 3. Determinant factors for VCT utilization by the students in Ambo University, March, 2012.

\begin{tabular}{llllll}
\hline \multirow{2}{*}{ Variables } & & \multicolumn{2}{l}{ VCT is Important } & \multirow{2}{*}{ COR(95\% CI) } & AOR(95\% CI) \\
\cline { 3 - 5 } & & Yes & No & & $3.82(1.53,9.54)^{*}$ \\
\hline Age & $15-20$ & $296(39.5)$ & $30(4.0)$ & $2.4(1.06,5.45)^{*}$ & $2.43(1.02,5.83)^{*}$ \\
& $21-25$ & $334(44.5)$ & $44(5.9)$ & $1.85(0.84,4.08)$ & 1 \\
Batch(year) & $>$ /=26 & $37(4.9)$ & $9(1.2)$ & 1 & $0.65(0.33,1.25)$ \\
& $1^{\text {st }}$ year & $231(30.8)$ & $35(4.7)$ & $0.67(0.36,1.23)$ & $1.01(0.51,1.99)$ \\
VCT ever used & $2^{\text {nd }}$ year & $268(35.7)$ & $31(4.1)$ & $0.88(0.47,1.63)$ & 1 \\
& $3^{\text {rd }}$ year & $168(22.4)$ & $17(2.3)$ & 1 & $2.12(1.27,3.54)$ \\
Having sexual partner & Yes & $461(61.5)$ & $43(5.7)$ & $27.8(13.93,55.51)^{*}$ & 1 \\
& No & $206(27.5)$ & $40(5.3)$ & 1 & $0.59(0.35,1.00)$ \\
\hline
\end{tabular}

*p-valve less than 0.05

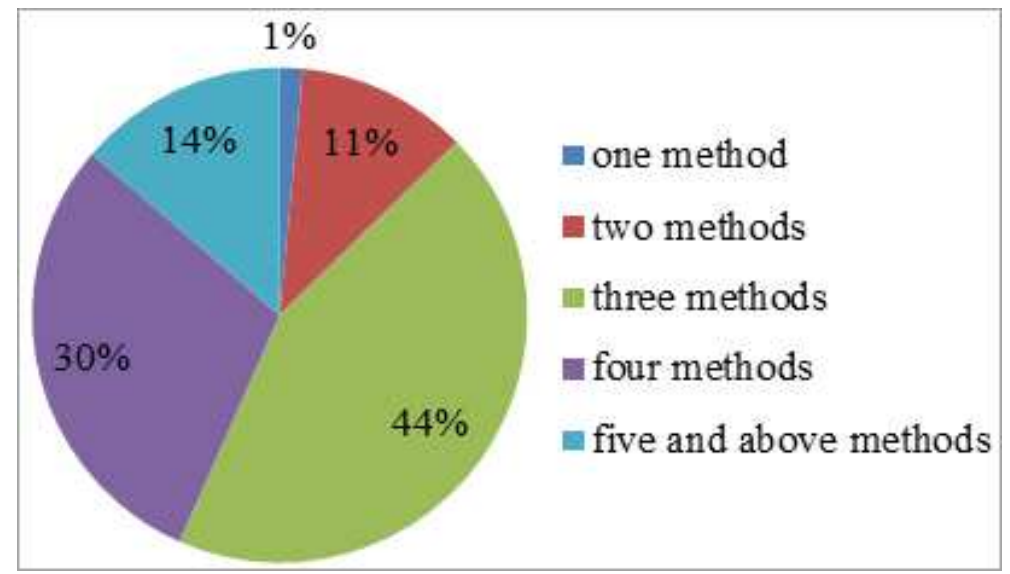

Figure 1. Numbers of HIV transmission methods known by the students, Ambo University March, 2012. 


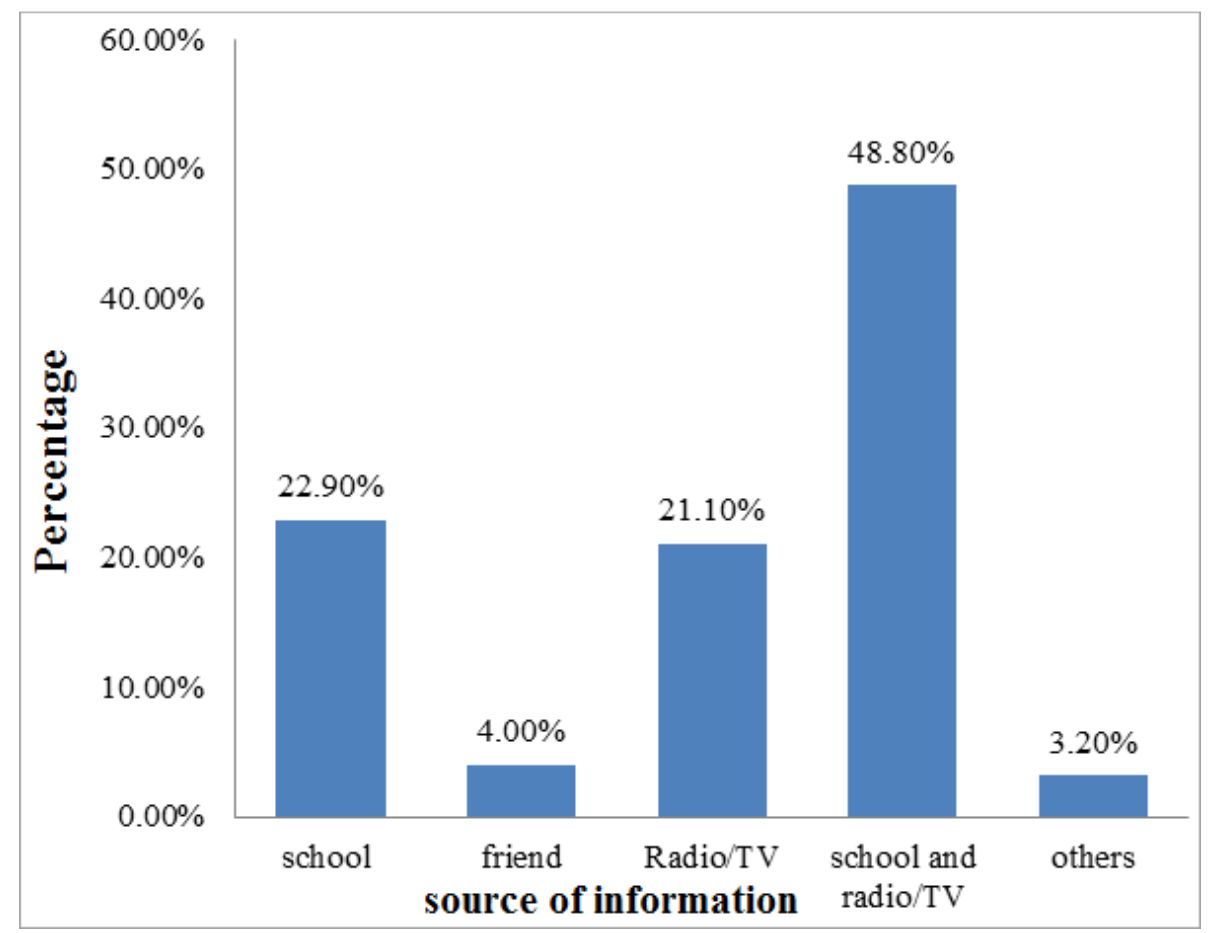

Figure 2. Source of information about HIV the students in Ambo University, March, 2012.

\section{Determinants of VCT Utilization}

There were different determinants those have statistical association with VCT service utilization include sex, batch, sexual exposure and knowledge of the students about VCT. The sex of the students had strong statistical association with the utilization of VCT service. Male students were found 33\% times less likely used VCT service when compared with female students. First year students were found 2.28 times higher utilized VCT service when compared with third year students. The knowledge of the students found to have strong statistical association with VCT utilization. Those who say yes for the importance of VCT found 2.18 times higher than those say no for the importance of VCT. Sexual experience was also found to have statistical association with VCT utilization (Table 3).

Age of the students, VCT service ever used and having sexual partners were the determinant factors statistically associated with the importance (knowledge) of the student about VCT. Students aged from 15-20 years old were 2.4 times more knowledgeable when compared to students aged greater or equal to 26 years of age. Those who ever used VCT 2.12 times higher reported as VCT is important when compared to students who did not ever used VCT service (table 4)

Table 4. Determinant factors for important of VCT utilization by the students in Ambo University, March, 2012.

\begin{tabular}{llllll}
\hline \multirow{2}{*}{ Variables } & & \multicolumn{2}{l}{ VCT Utilization } & \multirow{2}{*}{ COR(95\% CI) } & AOR(95\% CI) \\
\cline { 3 - 5 } & & Yes & No & & $0.61(0.42,0.90)^{*}$ \\
\hline sex & Male & $390(52.0)$ & $171(22.8)$ & $0.67(0.47,0.93)^{*}$ & 1 \\
Batch & Female & $114(15.2)$ & $75(10.0)$ & 1 & $0.90(0.59,1.39)$ \\
& First year & $179(23.9)$ & $87(11.6)$ & $2.28(1.36,3.83)^{*}$ & $1.06(0.69,1.62)$ \\
& Second year & $209(23.9)$ & $90(12.0)$ & $1.31(0.77,2.25)$ & 1 \\
Sexual experience & Third year & $116(15.3)$ & $69(9.2)$ & 1 & 1 \\
& Yes & $306(40.8)$ & $83(11.1)$ & 1 & $0.21(0.13,0.41)^{*}$ \\
Importance of VCT & No & $198(26.4)$ & $163(21.8)$ & $0.20(0.10,0.38)^{*}$ & $2.03(1.21,3.40)^{*}$ \\
& Yes & $461(61.5)$ & $206(27.5)$ & $2.18(1.32,3.64)^{*}$ & 1 \\
\hline
\end{tabular}

*p-valve less than 0.05

\section{Discussion}

This study tried to gather different information about VCT services from the students on the hope that it will help to show their point of view on the existing services and how to make it more accessible so as to serve these target group better.

In this study $389(51.9 \%)$ of the respondents had sexual experience, or ever had sexual intercourse; out of which 288 $(38.4 \%)$ were males and the rest $101(13.5 \%)$ were females. 
These figures are higher than the figures recorded by the study in Shanghai which was $18.8 \%$ male and $16.8 \%$ female students had sexual experience. At the time of this survey period $44.3 \%$ of the students had one or more sexual partners. Of which $31.7 \%$ were males and $12.5 \%$ were females. Among those had sexual experience $86.1 \%$ them were started at age group of $14-19$ years. Of this $61.2 \%$ were males and $24.9 \%$ were females. The findings of this study revealed that majority of the students were experienced sexual practice at early age. Male students were more experienced early sexual practice than female students. This may be due to the students were separated from their parent and have less control as well for male students the less restricted culture of the society might contribute to start early sexual practice than female students.

Regarding the protective method for HIV, $46.9 \%, 26.0 \%$ and $27.2 \%$ of respondents were reported by abstinence, faithfulness and uses of condom respectively. This finding was lower than the finding of research done in Gondar high school students, abstinence; faithfulness to one's sexual partner uses of condom as means of preventing HIV was reported by $84.1 \%, 60.4 \%$ and $41.8 \%$ respectively. This might be due to variation of culture in these two study area, as well university students far away from their parents than high school students.

The majority of the students $(88.9 \%)$ were reported that VCT is important in the prevention and controlling of HIV transmission where as $11.1 \%$ of the students were believed that VCT had no important in the prevention and controlling of HIV transmission. The finding of this study was lower than the finding of a study done in Addis Ababa which was $100 \%$ of the study participants were reported VCT is important in the prevention and controlling of HIV transmission. This variation might be due to information availability and accessibility variation; information more accessible and available in the capital city of Ethiopia (Addis Ababa) than Ambo (study area).

Male students were found less likely used VCT service when compared with female students and first year students were more utilized VCT service when compared with third year students. The reason for this might be male students may perceive as they are less risk or more relaxant.

\section{Conclusion}

Even if majority of the students were reported as VCT is important for HIV prevention still there were some students who did not know the important of VCT service in the prevention of HIV transmission. More than half of the participants were unable able to mention the protective measure taken to prevent HIV transmission. Most of the students had early sexual practice. More than half of the participants were used VCT service as well as have positive attitude towards VCT utilization. Sex, sexual exposure and their level of education (batch), age and the presence of sexual partner were some of the determinant factors identified. Remarkable numbers of students were recommended to established VCT service in the university. So it is important to establish VCT center in the university and doing further research.

\section{References}

[1] Helen Jackson. AIDS Africa- continent in crises, SIDA, UNPPA, SAFIDS 2001.

[2] UNAIDS. Voluntary counseling and testing Technical up date, UNAIDS best practice collection. May 2000, Geneva Switzerland.

[3] Equitable access to HIV counseling and testing for youth in developing countries: A review of current practice. Washington, DC: Population council. Horizon Accessed June 4-10- 2006.

[4] FHI. Voluntary counseling and testing and young people a summary overview, June 2002.

[5] UNAIDS. Report on the global AIDS epidemic 4th global report, 6 July 2004 Geneva Switzerland.

[6] Max Essex.et.al. AIDS in Africa second edition, New York, 2002.

[7] Balmer D, Grinstead O, et al. Cost effectiveness of voluntary counseling and testing in reducing sexual transmission of HIV-1 in Kenya and Tanzania, the Lancet 2000;356:113-21.

[8] Gibier de souza. L, Verga A, Cardoso J, ManjateRM, Barereto A. Using MOH-NGO partnership to implement VCT in Mozambique, IAS, 14thInternational AIDS Conference Barcelona, 2002

[9] UNADIS, 2006, report on the global ADIS epidemic, the impact of ADIS on people and societies

[10] DeGraft Johnson, Joseph et al. HIV voluntary counseling and testing service preferences in a Rural Malawi population. AIDS and Behavior, volume 9, Number 4, Dec 2005, pp. 475 $-484,(10)$ pub med.

[11] Nigatu R, Seman K. Attitudes and practices on HIV preventions among students of higher education institutions in Ethiopia: The case of Addis Ababa University, Educational Research 2011,Vol. 2(2)

[12] Anuar Yibrie. Determinants of HIV-VCT Acceptance in Gondar Town, North West Ethiopia, Master thesis Nov 2006 A.A Ethiopia.

[13] Alemayehu Bahray, Assessment of KAP on VCT among Mekele University students, MEJS Volume 2 (1): 108-118, 2010

[14] Tsehaye T, Bikes D, Mengesha A, Bayeh A, Assessment of factors associated with voluntary counseling and testing uptake among students in Bahir Dar University: Ethiop. J. Health Dev.2012;26(1):16-21 